\title{
INCIDENCIA DEL DETERIORO PROGRESIVO DEL ARBOLADO URBANO EN EL VALLE DE ABURRÁ, COLOMBIA
}

\section{Incidence of progressive deterioration of urban trees in the Aburrá valley, Colombia}

\author{
Héctor Iván Restrepo O. ${ }^{1}$, Flavio Moreno H. ${ }^{2}$ \& Claudia Helena Hoyos E. ${ }^{3}$
}

Restrepo O., H. I.; Moreno H., F. \& Hoyos E., C.H. (2015). Incidencia del deterioro progresivo del arbolado urbano en el Valle de Aburrá, Colombia. Colombia Forestal, 18(2),225-240.

Recepción: 17 de diciembre de 2014

\section{RESUMEN}

El arbolado urbano de los municipios del Valle de Aburrá, Colombia, está evidenciando deterioro y muerte por estrés hídrico y térmico, contaminación atmosférica, urbanización y ataques de plagas y enfermedades. Se realizó un muestreo de 11710 individuos de 25 especies de árboles y palmas en el área metropolitana del Valle de Aburrá, con el propósito de registrar síntomas de deterioro progresivo (DP) como marchitez de ramas y secamiento descendente. Se estimó un modelo logit multifactorial que relaciona la incidencia del DP con características dasométricas de los individuos, el sitio de siembra y el número de afectaciones. Se encontraron 720 individuos con DP (incidencia de 6.1\%). Los modelos logit tuvieron un ajuste satisfactorio a los datos y evidenciaron que existe una relación estadísticamente significativa entre la incidencia del deterioro de árboles y el diámetro del árbol, su ubicación (municipio), especie, sitio de siembra (zona verde, piso duro, alcorque) y presencia de otras afectaciones.

Palabras clave: área metropolitana del Valle de Aburrá, modelos logit, secamiento descendente, silvicultura urbana.
Aprobación: 13 de abril de 2015

\begin{abstract}
The urban forest of the municipalities of the Aburra Valley, Colombia, is deteriorating as a result of drought and heat stress, air pollution, urbanization, pests and diseases. In order to record the symptoms of progressive deterioration $(D P)$ of trees such as wilting of branches and downward drying, a sample of 11710 individuals from 25 tree species and palms in the metropolitan area of the Aburrá Valley was carried out. A multivariate logit model was estimated to relate the incidence of $D P$ with dasometric characteristics of individuals, the planting site and the extent of damage. 720 individuals with DP (incidence $6.1 \%$ ) were found. The logit model had a satisfactory fit to the data and showed that there is a statistically significant relationship between the incidence of tree deterioration and tree diameter, location (municipality), species, planting site (green, hard floor, container ) and other damaging factors.
\end{abstract}

Keywords: metropolitan area of the Aburrá Valley; logit models; downward drying; urban forestry.

1 Universidad Nacional de Colombia. Calle 59a 63-020, Autopista Norte, bloque 20, oficina 212. Medellín, Colombia. Código postal 050034.hirestrepoo@unal.edu.co. Autor de correspondencia

2 Universidad Nacional de Colombia. Calle 59a 63-020, Autopista Norte, bloque 20, oficina 212. Medellín, Colombia. Código postal 050034.fhmoreno@unal.edu.co

3 Área Metropolitana del Valle de Aburrá, Carrera 53 № 40A-31 Medellín, Colombia. Código postal 050034. claudia.hoyos@metropol.gov.co 


\section{INTRODUCCIÓN}

El deterioro de la salud de un árbol y su eventual muerte es un fenómeno complejo en el cual interactúan varios factores, los cuales se suelen clasificar en abióticos y bióticos. Entre los primeros se pueden mencionar el fuego (incluyendo los rayos), contaminación atmosférica, viento, erupciones volcánicas y estrés ambiental, el cual es producido por diversas causas, entre ellas el cambio climático. Entre las causas bióticas se pueden mencionar senescencia, desequilibrio mecánico, inanición, respiración, herbivoría, enfermedades, plagas y competencia (Waring, 1987). Las causas bióticas se suelen subdividir a su vez en endógenas y exógenas (Franklin et al., 1987). Sin embargo, estas clasificaciones no alcanzan a representar adecuadamente las interacciones entre los árboles, su ambiente y los agentes deletéreos de su salud, las cuales a su vez varían en el espacio y en el tiempo. La variación espacial puede expresarse a escala local (por ejemplo, de rodal o pequeñas secciones de bosque) o más amplia (de paisaje); la incidencia temporal se puede dividir a su vez en episódica, gradual o progresiva y súbita (Lugo \& Scatena, 1996). En general, independientemente de las causas, los árboles mueren cuando no logran metabolizar recursos suficientes que garanticen un mínimo vital, especialmente cuando ocurren sequías o deficiencia de nutrientes, lo cual conduce a una mayor susceptibilidad al ataque de insectos y enfermedades (Waring, 1987).

Además del cambio climático, el cual es considerado como uno de los principales factores responsables de la alteración de los ecosistemas forestales (Spittlehouse \& Stewart, 2003; Dale, et al., 2010), el arbolado urbano es especialmente vulnerable a otros factores ambientales distintos del cambio climático, pues en las ciudades los árboles suelen estar sometidos a condiciones desfavorables relacionadas con la contaminación, el estrés térmico producido por las islas de calor y el estrés hídrico derivado de la impermeabilidad de los suelos urbanos, entre otros. Todo ello provoca desbalances fisiológicos que aumentan la susceptibilidad al ataque de plagas y enfermedades y, finalmente, su muerte (Cregg \& Dix, 2001). El factor antrópico también suele incidir en la salud de los árboles: en forma pasiva por la planeación deficiente del establecimiento de los árboles en la ciudad y, en forma activa, por maltrato, cortes indebidos, anillamiento, envenenamiento, quemas, etc.

El presente estudio se motivó por la aparición de síntomas aparentes de muerte súbita de árboles en las zonas verdes urbanas de los nueve municipios que conforman el área metropolitana del Valle de Aburrá. Un fenómeno similar ha sido registrado en otros lugares del mundo y se caracteriza por la muerte repentina de rodales o bosques (Waring, 1987; Rizzo \& Garbelotto, 2003; Santamour Jr., 2004; Guo et al., 2005; Lacan \& McBride, 2008; Garbelotto \& Schmidt, 2009; Sturrock et al., 2011). Sin embargo, en el Valle de Aburrá no se ha presentado una devastación con muerte repentina como la ocurrida en otros países, por lo cual el término "deterioro progresivo" (DP) es probablemente más apropiado para denominar dicho fenómeno. El objetivo de este trabajo fue analizar la magnitud de la incidencia del DP en zonas verdes urbanas del Valle de Aburrá y su relación con algunas variables endógenas y exógenas, entre ellas la especie, tamaño de los árboles, localización geográfica, características del sitio de siembra e historia del manejo (evaluada mediante el número de afectaciones sufridas durante el desarrollo de los árboles). Se espera que este análisis sea de utilidad para la planificación del arbolado urbano con criterios técnicos, la reducción de los costos para su mantenimiento y la mitigación del riesgo asociado al deterioro progresivo de árboles ubicados en zonas urbanas de alto flujo peatonal y vehicular.

\section{METODOLOGÍA}

\section{Área de estudio}

La presente investigación se realizó en la zona urbana del área metropolitana del Valle de Aburrá, la cual comprende una conurbación de nueve 
municipios: Medellín, Bello, Itagüí, Sabaneta, La Estrella, Caldas, Copacabana, Girardota y Barbosa, en una extensión de $320 \mathrm{~km}^{2}$, con centro en la ciudad de Medellín, capital del departamento de Antioquia, Colombia (Figura 1). El rango altitudinal del área urbana va de 1300 metros de altitud

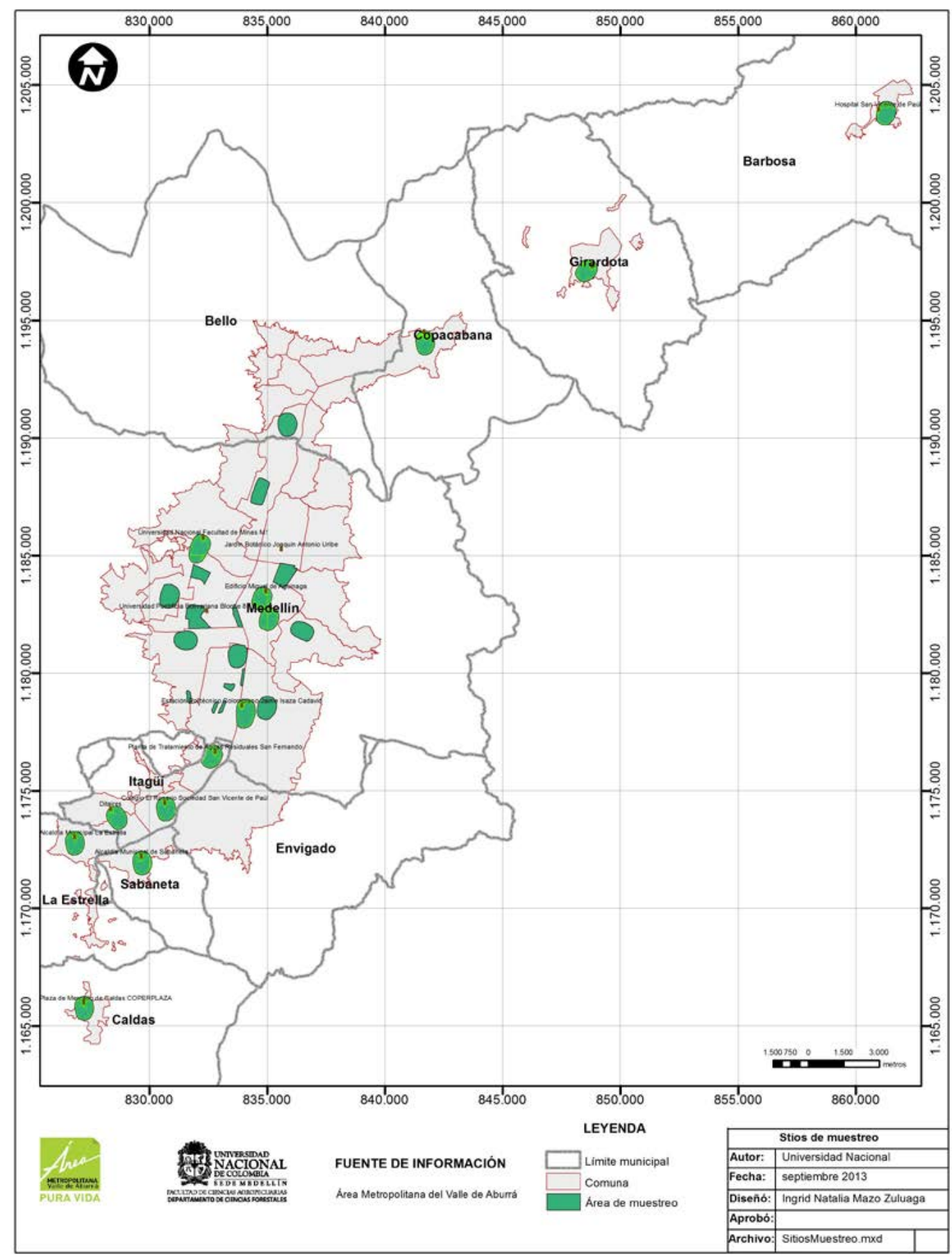

Figura 1. Zonas de muestreo para la evaluación de la magnitud del deterioro progresivo del arbolado urbano en el Área Metropolitana del Valle de Aburrá. 
en Barbosa a 1800 metros de altitud en La Estrella; de manera similar, las temperaturas medias oscilan entre 24 y $20^{\circ} \mathrm{C}$, respectivamente (Corantioquia, 1997; Cardona, 2006). La precipitación tiene una distribución bimodal, con dos períodos lluviosos en marzo-mayo y en septiembre-noviembre; en la ciudad de Medellín, la precipitación media anual es de 1500 mm, la que aumenta ligeramente tanto hacia el sur como hacia el norte, por lo cual Caldas y Barbosa son los municipios más Iluviosos, donde es superior a los 2000 mm (AMVA \& UNAL, 2014). Las condiciones ecológicas de la mayoría del área de estudio corresponden a la zona de vida bosque húmedo premontano ( $\mathrm{Hol}$ dridge, 1996), excepto en los municipios de Barbosa y Caldas, que corresponden a bosque muy húmedo premontano. Existe una amplia variabilidad en los suelos, producto de una geomorfología compleja que varía ampliamente en un valle estrecho como el del río Medellín (AMVA et al., 2007). Quizás la característica común más sobresaliente es el profundo deterioro de los suelos resultante del proceso de urbanización, por lo cual estos suelen ser compactados y mal drenados, con presencia abundante de desechos de construcción que ofrecen limitaciones para el adecuado crecimiento de los árboles (AMVA et al., 2010; AMVA \& UNAL, 2014).

\section{Mediciones de campo}

La magnitud del DP de los árboles urbanos en el Valle de Aburrá se evaluó en 29 zonas representativas, donde se midió una muestra estadísticamente significativa de las 25 especies de árboles y palmas con mayor abundancia relativa (Tabla 1) reportadas por el Plan Maestro de Espacios Públicos Verdes Urbanos del área metropolitana del Valle de Aburrá (AMVA, 2007). El presente análisis se realizó únicamente en árboles plantados en el espacio público verde, por lo cual no se realizaron muestreos en bosques naturales que quedaron englobados por las zonas urbanas.
A cada uno de los árboles y palmas (individuos) muestreados de las 25 especie estudiadas se le asignó un único número de identificación, al cual se le asoció información espacial, dasométrica, dendrológica, estado fitosanitario y características silviculturales. Se registró la circunferencia a 1,3 $\mathrm{m}$ de altura con cinta métrica (precisión de $1 \mathrm{~mm}$ ), la cual fue luego transformada en diámetro (conocido como diámetro a la altura del pecho, DAP) y altura total $(\mathrm{H})$ con hipsómetro Suunto ${ }^{\circledR}$, así como la identificación de la especie (Sp).

Para evaluar el fenómeno de DP, se registró la ausencia o presencia de marchitez de ramas y secamiento descendente en tres grados o niveles de severidad: bajo (afecta a menos del $20 \%$ de la copa), medio (afecta entre 20 y $60 \%$ de la copa) y alto (afecta más del 60\% de la copa). El secamiento descendente se caracteriza por la marchitez de las ramas superiores, cuya primera manifestación es la caída de sus hojas y la muerte de yemas terminales; eventualmente este mal se propaga a toda la copa del árbol hasta las ramas bajas, con defoliación asincrónica, persistente, generalizada y muerte del árbol. También se contabilizó el número de afectaciones (\#A) que aparecían simultáneamente en el mismo árbol (\#A es un número entero de 0 a 7), así como las afectaciones que resultaron evidentes. Las más importantes fueron: daños mecánicos, ataque de plagas, hongos, espacio reducido, vandalismo, anillamiento y quemas. Una última variable registrada fue el sitio de siembra (Ss) de los árboles, el cual se dividió en tres categorías previamente establecidas: piso duro, que corresponde a los árboles ubicados en zonas contiguas a andenes y vías pero sin contenedor de raíces; alcorque, que corresponde a árboles sembrados en contenedores de raíces; y zona verde, que corresponde a los árboles sembrados en zonas verdes amplias como parques, intercambios viales y glorietas, donde aparentemente no existe restricción para el desarrollo de raíces. Todas las mediciones de campo se realizaron entre noviembre de 2012 y febrero de 2013. 
Tabla 1. Información de las especies muestreadas para DP en el área metropolitana del Valle de Aburrá. DAP: diámetro normal $(\mathrm{cm}), H$ : altura $(\mathrm{m}), n$ : número de individuos afectados, $N$ : número total de individuos, $l=n / N$ : incidencia

\begin{tabular}{|c|c|c|c|c|c|}
\hline Especies & DAP Promedio & $H$ Promedio & $\mathbf{n}$ & $\mathbf{N}$ & $I$ \\
\hline Archontophoenix cunninghamiana & 17.4 & 6.2 & 1 & 884 & 0 \\
\hline Bauhinia picta & 30.4 & 9 & 35 & 293 & 0.12 \\
\hline Caesalpinia peltophoroides & 28.9 & 6.9 & 62 & 1031 & 0.06 \\
\hline Callistemon speciosus & 18.8 & 5.8 & 5 & 140 & 0.04 \\
\hline Cordia alliodora & 27.5 & 11.1 & 3 & 55 & 0.05 \\
\hline Eriobotrya japonica & 9.2 & 3.4 & 1 & 382 & 0 \\
\hline Erythrina fusca & 42.2 & 9.5 & 5 & 383 & 0.01 \\
\hline Eucalyptus sp. & 51.3 & 17.3 & 4 & 288 & 0.01 \\
\hline Ficus benjamina & 43.2 & 8.8 & 246 & 1295 & 0.19 \\
\hline Fraxinus chinensis & 46.9 & 11.3 & 11 & 1090 & 0.01 \\
\hline Jacaranda mimosifolia & 36.1 & 9.9 & 12 & 368 & 0.03 \\
\hline Lafoensia punicifolia & 22.4 & 8.6 & 30 & 245 & 0.12 \\
\hline Lagerstroemia speciosa & 20.4 & 6.1 & 12 & 251 & 0.05 \\
\hline Melaleuca quinquenervia & 23.9 & 7.2 & 8 & 168 & 0.05 \\
\hline Melicoccus bijugatus & 33.9 & 8.5 & 4 & 74 & 0.05 \\
\hline Ochroma pyramidale & 40.5 & 9.9 & 0 & 12 & 0 \\
\hline Pithecellobium dulce & 28.9 & 8.1 & 84 & 504 & 0.17 \\
\hline Pithecellobium longifolium & 19.7 & 7.4 & 11 & 293 & 0.04 \\
\hline Psidium guajava & 14 & 4.9 & 16 & 560 & 0.03 \\
\hline Roystonea regia & 48.4 & 9.8 & 2 & 452 & 0 \\
\hline Spathodea campanulata & 34.3 & 8.7 & 80 & 856 & 0.09 \\
\hline Syagrus romanzoffiana & 29.3 & 7.7 & 11 & 307 & 0.04 \\
\hline Syzygium malaccense & 18.1 & 6.2 & 6 & 495 & 0.01 \\
\hline Tabebuia chrysantha & 18.2 & 6.5 & 27 & 600 & 0.05 \\
\hline Terminalia catappa & 25.1 & 7.4 & 44 & 684 & 0.06 \\
\hline Total & 30.4 & 8.1 & 720 & 11710 & 0.06 \\
\hline
\end{tabular}

\section{Cálculo de la incidencia del DP}

Los datos provenientes de las mediciones de campo se usaron para calcular la tasa de incidencia (ecuación 1) del DP de los árboles (Cooke, 2006).

$$
I=\frac{y}{Y}
$$

Donde: I, tasa de incidencia; $y$, número de individuos con síntomas de $D P$ y $Y$, número total de individuos evaluados (sanos + afectados). Con los datos de la tasa de incidencia se calculó el error de muestreo según la ecuación 2 (Walpole \& Myers, 1992).

$$
e=\sqrt{\frac{Z_{\propto / 2}^{2} \tilde{P}(1-\tilde{P})}{n}}
$$


Donde: e, error de muestreo; $Z_{\propto / 2^{\prime}}^{2}$ distribución normal estándar; $\widetilde{P}$, proporción o, en este caso, incidencia; $n$, número de árboles medidos. Así mismo, los valores de tasa de incidencia de DP se agruparon en 4 categorías: i) muy severo $(15-20 \%)$, ii) severo (10-15\%), iii) moderado (5-10\%) y iv) bajo ( $<5 \%)$.

\section{Determinantes del DP}

Con base en las variables analizadas, se estimó un modelo logit para determinar la relación de variables independientes o factores con la incidencia del DP de zonas verdes árboles urbanas. El logit permite analizar fenómenos cuya variable dependiente es limitada y, particularmente, de respuesta dicotómica o binaria (Wooldridge, 2002; Cooke, 2006), que en este caso particular corresponde a la presencia o no de síntomas de DP. Los modelos logit se definen matemáticamente según la ecuación 3, o en su forma lineal según la ecuación 4.

$$
\begin{aligned}
& P=\frac{e^{\beta_{0} X_{0}+\beta_{1} X_{1}+\beta_{2} X_{2}+\ldots \beta_{n} X_{n}}}{1+e^{\beta_{0} X_{0}+\beta_{1} X_{1}+\beta_{2} X_{2}+\ldots \beta_{n} X_{n}}} \\
& \ln \left(\frac{p}{1-p}\right)=e^{\beta_{0} X_{0}+\beta_{1} X_{1}+\beta_{2} X_{2}+\ldots \beta_{n} X_{n}}= \\
& =\sum_{i=0}^{n} \beta_{i} X_{i}
\end{aligned}
$$

Donde: $p$, probabilidad de ocurrencia del fenómeno para un nivel de $x_{i}$ dado; $\beta_{i}$ parámetros a estimar; $x_{i}$ variables exógenas o independientes con $x_{0}=1$; e, base de los logaritmos naturales y In, logaritmo natural. Para cada uno de los parámetros $\beta_{i}$ se estimó además el intervalo de confianza del 95\%. Para el presente estudio, este modelo logit multifactorial estimado (ecuaciones 3 y 4 ), se puede escribir como:

$$
P=\frac{e^{\beta_{0}+\beta_{1} \cdot D a p+\beta_{2} \cdot M p i o+\beta_{3} . S p+\beta_{4} \cdot S s+\beta_{5} . \# A}}{1+e^{\beta_{0}+\beta_{1} \cdot D a p+\beta_{2} \cdot M p i o+\beta_{3} \cdot S p+\beta_{4} \cdot S s+\beta_{5} . \# 5}}
$$

$\mathrm{O}$

$$
\ln (p / 1-p)=\beta_{0}+\beta_{1} \cdot \text { Dap }+\beta_{2} . \text { Mpio }+\beta_{3} . S p+\beta_{4} . S s+\beta_{5} . \# 5
$$

Donde $D A P$ es el diámetro a la altura del pecho (cm), Mpio es el municipio donde está ubicado el árbol, $S p$ corresponde a la especie del árbol, SS corresponde a las categorías de sitio de siembra, \#A es el número de afectaciones que tenía árbol (es un número entero que va de 0 a 7) y $\beta_{i}$ son los parámetros estimados. En dicho modelo hay una variable independiente numérica continua y una discreta, $D A P$ y $\# A$, respectivamente, y tres variables tipo factor: Mpio, $S p$ y $S$ s. Debido a la estructura de los modelos logit, el intercepto corresponde a valores nulos en variables cuantitativas (valores de cero para $D A P$ y \#A) y primer nivel de variables definidas como factor. En este último tipo de variables, el factor no es jerárquico en las variables Mpio y $S p$, ya que no hay posibilidad de identificar a priori el efecto de la ubicación o especie en el DP, mientras que el factor es jerárquico en $S s$, ya que se supone que los árboles sembrados en zonas verdes amplias tendrían una incidencia menor de DP que los sembrados en alcorque, y estos a su vez, menor incidencia de DP que los sembrados en piso duro.

Aunque este modelo puede ser usado para predecir, en este trabajo se usó principalmente para explicar el efecto de las variables independientes sobre el DP de árboles en zonas verdes urbanas. Para verificar la significancia estadística del modelo, se aplicó la prueba de Wald (Griliches \& Intriligator, 1994), la cual consiste en determinar el aporte a la varianza de cada uno de los factores incluidos en el modelo.

Se elaboraron histogramas de frecuencias del número total de individuos y del número de individuos con síntomas de deterioro progresivo por clases de diámetro y altura; así mismo, se graficó la proporción de árboles con síntomas de DP por clase de diámetro y por número de afectaciones. Estas proporciones se calcularon con la ecuación 7. 


$$
P a_{i}=\frac{y_{i}}{Y_{i}^{\prime}}
$$

Donde: $P a_{i}$ proporción de individuos afectados en la clase de diámetro $i ; y_{i}$, número de individuos afectados en la clase $i ; Y_{i^{\prime}}$ número de individuos en la clase de diámetro $i$. Todos los análisis estadísticos se realizaron en R (R Core Team, 2013).

\section{RESULTADOS}

\section{Datos dasométricos}

El error de muestreo alcanzado con el número de árboles muestreados (ecuación 2) fue de 0.00437, o 7.1\%. Las frecuencias de los árboles y palmas muestreados con respecto al diámetro y a la altura presentaron distribuciones unimodales con asimetría hacia la derecha (Figuras 2 y 3). La clase diamétrica modal $(12.5-25 \mathrm{~cm})$ tuvo una proporción de individuos de $32 \%$ respecto al total (Figura 2). En el histograma de alturas, tres clases (entre 2.5 y $10 \mathrm{~m}$ ) representaron el $70 \%$ de los árboles y palmas muestreados del Valle de Aburrá (Figura 3).

\section{Incidencia del DP}

Se encontraron 720 árboles y palmas con síntomas de DP, que equivalen a una tasa de incidencia de $6.1 \%$ (Tabla 1), la cual es estadísticamente

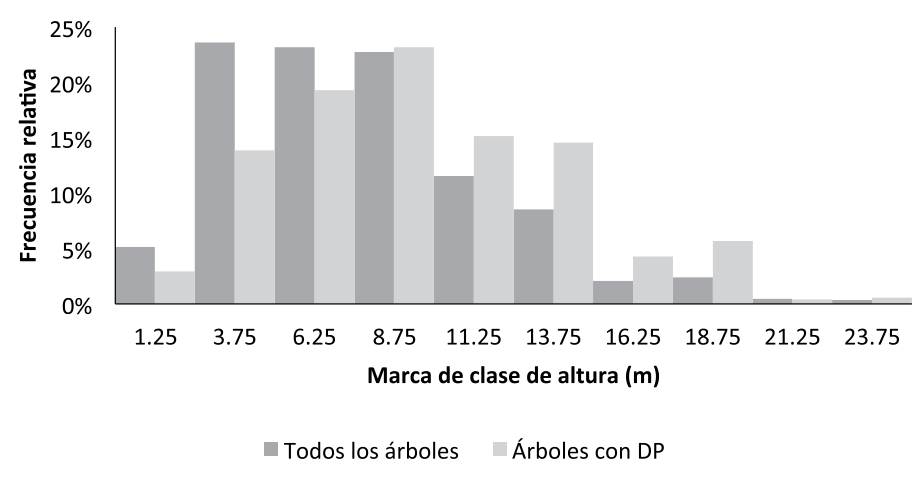

Figura 2. Histograma de frecuencias relativas de árboles y palmas por clases de diámetro para toda la muestra (11 710 individuos en total) y para los individuos con deterioro progresivo (DP) (720 individuos en total).

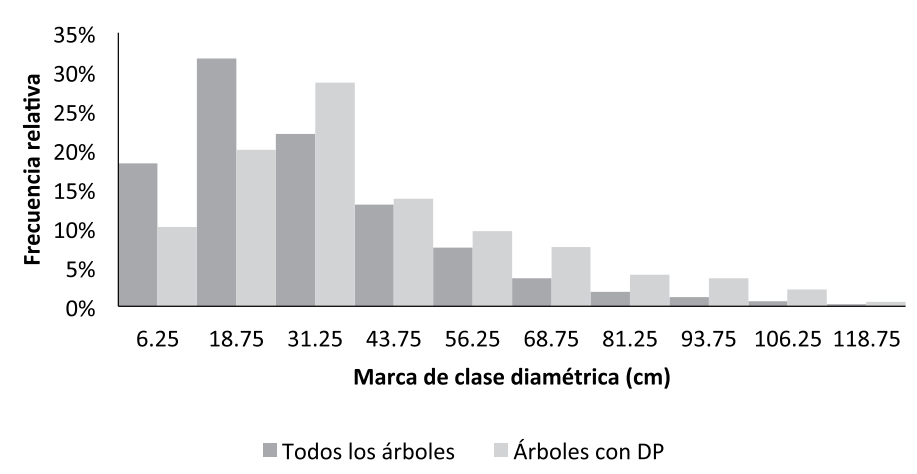

Figura 3. Histograma de frecuencias relativas de árboles y palmas por clases de altura para toda la muestra (11 710 individuos en total) y para los individuos con deterioro progresivo (DP) (720 individuos en total). 
diferente de cero $(\mathrm{p}<0.05)$. La distribución de frecuencias por clases de diámetro y de altura de los árboles que presentaron DP fue similar a la de la muestra de árboles estudiada (Figuras 2 y 3); no obstante, la moda de las clases diamétricas de los árboles con DP fue mayor (marca de clase de $31.25 \mathrm{~cm}$, en la cual se presentó el $28 \%$ de los individuos) que para los individuos muestreados (marca de clase de $18.75 \mathrm{~cm}$ ). Lo mismo ocurrió con la distribución de las alturas de los individuos con DP, cuyo valor modal de 8.75 m (que correspondió al 25\% de los árboles con DP) fue mayor que para los individuos muestreados (marca de clase de $3.75 \mathrm{~m}$ ). Tanto para la distribución de diámetros como de alturas, la comparación de las frecuencias relativas de los individuos con DP y de la muestra hace evidente que la frecuencia de los individuos con DP aumentó de manera progresiva en los individuos de mayor tamaño (Figuras 2 y 3 ). Así mismo, en los individuos de menor tamaño, la incidencia de DP fue proporcionalmente menor. Sin embargo, estos resultados o no significan que el DP haya aumentado en las especies con DAP promedio mayor (Tabla 1), pues el coeficiente de correlación de Pearson entre la incidencia de DP y el $D A P$ o altura promedio de las especies fue muy bajo (en ambos casos el valor de $r$ fue menor de 0.03) y no significativo.

Los resultados obtenidos muestran que se presentaron diferencias significativas en el nivel de incidencia de DP entre municipios; por ejemplo, fue más marcado en los municipios de Barbosa y Sabaneta, y menor en Girardota y La Estrella; en este último fue prácticamente cero (un individuo de 251 muestreados). En Medellín se encontraron 528 individuos con DP de 8713 individuos muestreados, equivalente a una incidencia de DP de $6.1 \%$, la cual es estadísticamente diferente de cero $(\mathrm{p}<0.05)$.

De acuerdo con la agrupación de los valores de incidencia de DP obtenidos para cada una de las especies, los resultados son: i) muy severo (15-20\%) en falso laurel (Ficus benjamina) y chiminango
(Pithecellobium dulce); ii) severo (10-15\%) en casco de vaca (Bauhinia picta) y guayacán de Manizales (Lafoensia punicifolia); iii) moderado (5-10\%) en acacia amarilla (Caesalpinia peltophoroides), nogal (Cordia alliodora), flor de la reina (Lagerstroemia speciosa), corcho (Melaleuca quinquenervia), mamoncillo (Melicoccus bijugatus), tulipán africano (Spathodea campanulata), guayacán amariIlo (Tabebuia chrysantha) y almendro (Terminalia catappa); y iv) bajo $(<5 \%)$ en palma payanesa ( $A r$ chontophoenix cunninghamiana), calistemo rojo (Callistemon speciosus), níspero del Japón (Eriobotrya japonica), búcaro (Erythrina fusca), eucalipto (Eucalyptus sp.), urapán (Fraxinus udhei), gualanday (Jacaranda mimosifolia), balso (Ochroma pyramidale), suribio (Pithecellobium longifolium), guayabo (Psidium guajava), palma real de Cuba (Roystonea regia), palma de azúcar (Syagrus romanzoffiana) y pero de agua (Syzygium malaccense).

\section{Determinantes del DP evaluados en el modelo logit}

Intercepto (nivel base): en el modelo estimado, el intercepto corresponde al $D P$ de un individuo de $D A P=0$ en el municipio de Barbosa y de la especie palma payanesa (Archontophoenix cunninghamiana), plantada en una zona verde y sin afectaciones.

Tamaño: el valor del coeficiente estimado para $D A P(0.018)$ y el hecho de que su intervalo de confianza del 95\% (0.015-0.021) no incluya el valor de cero indican que esta variable es estadísticamente significativa $(p=0.001)$ y que a medida que se incrementa el diámetro, se incrementa también la probabilidad de sufrir deterioro progresivo.

Localización: con respecto al municipio donde se encuentran ubicados los árboles y palmas, no se encontró diferencia estadística entre el intercepto (Barbosa) y Bello, Caldas, Copacabana, Itagüí y Sabaneta; por el contrario, fueron estadísticamente diferentes al intercepto los ubicados en Girardota $(p=0.1)$, La Estrella $(p=0.01)$, Medellín $(p=0.05)$ y Parque de las Aguas $(p=0.1)$. 
Tabla 2. Coeficientes, intervalos de confianza y significancia estadística de las variables incluidas en el modelo logit de efectos múltiples de deterioro progresivo de árboles en zonas verdes urbanas en el Valle de Aburrá.

\begin{tabular}{|c|c|c|c|c|c|}
\hline \multirow{2}{*}{ Factor } & \multirow{2}{*}{ Efecto } & \multirow{2}{*}{ Coeficiente } & \multicolumn{2}{|c|}{ Intervalo de Confianza } & \multirow{2}{*}{ Significancia } \\
\hline & & & $2.5 \%$ & $97.5 \%$ & \\
\hline \multirow{3}{*}{ Tamaño } & Intercepto & -6.86 & -8.88 & -4.831 & $* * *$ \\
\hline & DAP & 0.018 & 0.015 & 0.021 & $* * *$ \\
\hline & Bello & -0.48 & -1.13 & 0.170 & \\
\hline \multirow{7}{*}{ Municipios } & Caldas & -0.41 & -1.38 & 0.558 & \\
\hline & Copacabana & -0.35 & -1.10 & 0.395 & \\
\hline & Girardota & -1.22 & -2.49 & 0.044 & + \\
\hline & Itagüi & -0.37 & -0.95 & 0.209 & \\
\hline & La Estrella & -2.94 & -4.97 & -0.902 & $* *$ \\
\hline & Medellín & -0.52 & -1.03 & -0.009 & $*$ \\
\hline & Parque de las Aguas & 0.617 & -0.06 & 1.291 & + \\
\hline \multirow{25}{*}{$\begin{array}{l}\text { Especies } \\
\text { forestales }\end{array}$} & Sabaneta & 0.309 & -0.29 & 0.907 & \\
\hline & Bauhinia picta & 4.535 & 2.539 & 6.531 & $* * *$ \\
\hline & Caesalpinia peltophoroides & 3.653 & 1.672 & 5.633 & $* * *$ \\
\hline & Callistemon speciosus & 3.366 & 1.209 & 5.523 & $* *$ \\
\hline & Cordia alliodora & 3.747 & 1.458 & 6.036 & $* *$ \\
\hline & Eriobotrya japonica & 1.027 & -1.75 & 3.803 & \\
\hline & Erythrina fusca & 1.788 & -0.37 & 3.948 & \\
\hline & Eucalyptus sp. & 1.996 & -0.21 & 4.200 & + \\
\hline & Ficus benjamina & 4.730 & 2.759 & 6.701 & $* * *$ \\
\hline & Fraxinus chinensis & 1.668 & -0.39 & 3.723 & \\
\hline & Jacaranda mimosifolia & 2.983 & 0.935 & 5.031 & $* *$ \\
\hline & Lafoensia punicifolia & 4.839 & 2.837 & 6.841 & $* * *$ \\
\hline & Lagerstroemia speciosa & 3.709 & 1.660 & 5.758 & $* * *$ \\
\hline & Melaleuca quinquenervia & 3.591 & 1.498 & 5.683 & $* * *$ \\
\hline & Melicoccus bijugatus & 3.566 & 1.352 & 5.779 & $* *$ \\
\hline & Ochroma pyramidale & -8.03 & -502 & 486.153 & \\
\hline & Pithecellobium dulce & 4.926 & 2.948 & 6.905 & $* * *$ \\
\hline & Pithecellobium longifolium & 3.181 & 1.122 & 5.240 & $* *$ \\
\hline & Psidium guajava & 3.362 & 1.335 & 5.388 & $* *$ \\
\hline & Roystonea regia & 0.692 & -1.72 & 3.100 & \\
\hline & Spathodea campanulata & 4.227 & 2.250 & 6.204 & $* * *$ \\
\hline & Syagrus romanzoffiana & 3.414 & 1.359 & 5.468 & $* *$ \\
\hline & Syzygium malaccense & 2.327 & 0.205 & 4.449 & $*$ \\
\hline & Tabebuia chrysantha & 3.709 & 1.709 & 5.710 & $* * *$ \\
\hline & Terminalia catappa & 3.835 & 1.847 & 5.823 & $* * *$ \\
\hline \multirow{2}{*}{ Sitio de siembra } & Alcorque & 0.348 & 0.119 & 0.576 & $* *$ \\
\hline & Piso duro & 0.393 & 0.132 & 0.654 & $* *$ \\
\hline Afectaciones & Número & 0.301 & 0.178 & 0.424 & $* * *$ \\
\hline
\end{tabular}

Significancia: ${ }^{* *}=0.001 ; * *=0.01 ; *=0.05 ;+=0.1$ 
Especie: para evaluar el efecto de especie, debe compararse con la probabilidad de sufrir DP en la especie del intercepto (palma payanesa), la cual tiene una incidencia de cero. Hecho esto, se encontró que la incidencia no fue diferente de cero en el níspero del Japón (Eriobotrya japonica), búcaro (Erythrina fusca), urapán (Fraxinus udhei), balso (Ochroma pyramidale) y palma real de Cuba (Roystonea regia) ( $p>0.1$ ) y es estadísticamente diferente de cero en las demás especies $(p<0.1)$ (Tabla 2).

Sitio de siembra: se encontró que los árboles plantados en zonas verdes amplias tienen menor probabilidad de sufrir DP que los árboles sembrados en piso duro o en alcorque, los cuales tienen coeficientes estimados de $0.348(p=0.05)$ y 0.393 $(p=0.05)$, respectivamente. El coeficiente de zona verde corresponde al intercepto, como se mencionó arriba.

Número de afectaciones: se encontró que a medida que se incrementa el número de afectaciones, también lo hace la probabilidad de sufrir DP, con un coeficiente asociado igual a $0.301(p=0.001)$. Si bien algunos de los municipios y especies tuvieron un efecto no significativo, la prueba de Wald mostró que el efecto combinado de los factores fue estadísticamente significativo $y$, por tanto, todas las variables analizadas se deben dejar en el modelo (Tabla 3).

Tabla 3. Valores de chi cuadrado y probabilidad (prueba de Wald) para verificar la significancia de las variables explicativas del DP usadas en el modelo logit multifactorial en los árboles de zonas verdes urbanas del Valle de Aburrá.

\begin{tabular}{lll}
\hline \multicolumn{1}{c}{ Variable } & \multicolumn{1}{c}{$\boldsymbol{X}^{\mathbf{2}}$} & \multicolumn{1}{c}{ Valor $\mathbf{p}$} \\
\hline DAP & 114.7 & 0.00 \\
Municipio & 51.6 & 0.00 \\
Especie & 357.8 & 0.00 \\
Sitio & 14.4 & 0.00 \\
N. Afectaciones & 22.9 & 0.00 \\
\hline
\end{tabular}

\section{DISCUSIÓN}

El modelo logit estimado indica que al aumentar el $D A P$ se incrementa la probabilidad de sufrir DP; este resultado está plenamente soportado por los datos de frecuencia de árboles afectados por clase de diámetro en comparación con el total de árboles presentes en la clase de diámetro respectiva (Figura 2). Así mismo, el hecho de que los datos de afectación por clases de altura hayan presentado un comportamiento idéntico (Figura 3) muestra de manera contundente que la incidencia de DP aumenta en los árboles más grandes. Aunque en el presente estudio no se evaluó la mortalidad, la relación entre el deterioro y la muerte de los árboles se ha establecido de manera clara (Manion, 1991); así mismo, en bosques naturales se ha reportado que con el incremento en las tallas de los árboles se incrementa también su probabilidad de deterioro y muerte (Del Valle, 2002; Yepes et al., 2010), tal como se evidencia con los resultados del presente estudio (Figura 4). Aunque la mortalidad de los árboles urbanos es generalmente mayor en los árboles jóvenes, especialmente en los dos o tres primeros años debido al estrés de trasplante (Miller \& Miller, 1991), superada esta etapa, la probabilidad de muerte aumenta en los árboles de mayor diámetro del tronco, probablemente debido a que los árboles más grandes tienen menor masa dinámica y por lo tanto menor resiliencia frente a factores de disturbio (Koeser et al., 2013).

Puesto que el tamaño de los árboles aumenta con la edad, es claro que la probabilidad de DP es mayor a medida que los árboles envejecen, no solo por la menor proporción de masa dinámica mencionada atrás, sino por el envejecimiento tisular (Salisbury \& Ross, 1985). Sin embargo, no se dispone de información de edades para evaluar en términos cuantitativos el efecto diferencial del envejecimiento sobre el DP de cada especie. Está bien establecido que la vida media de los árboles urbanos es menor que en bosques naturales, debido a los múltiples factores de estrés presentes en 


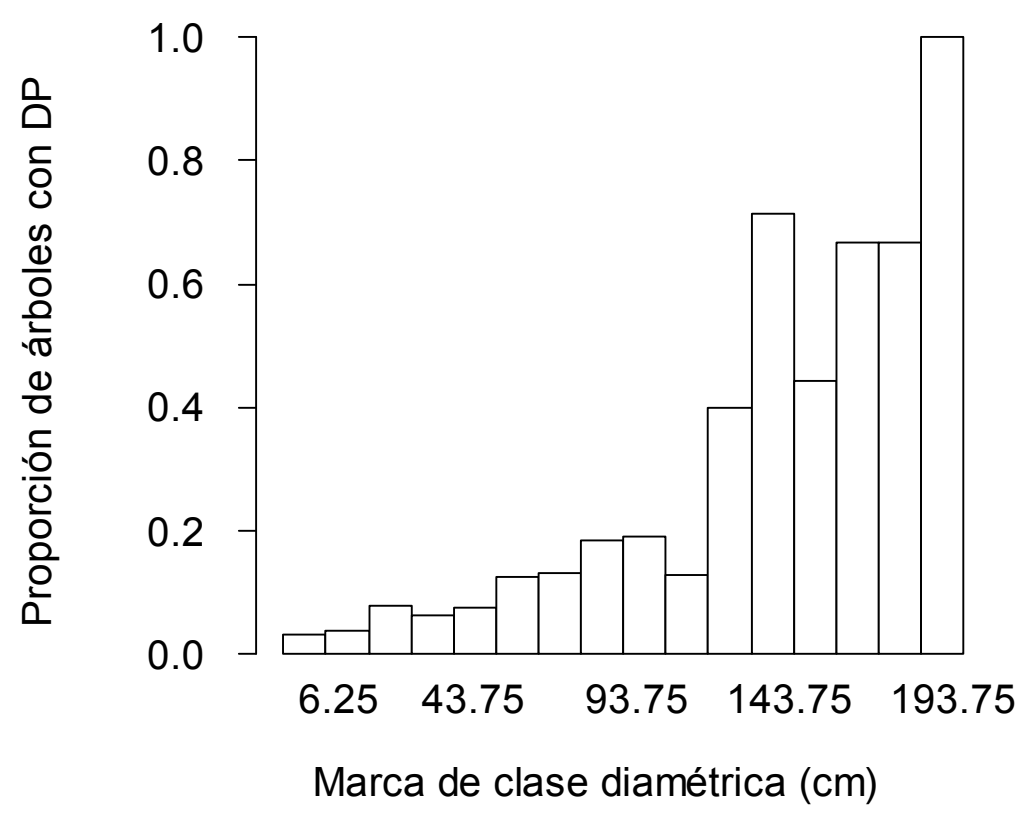

Figura 4. Proporción de árboles con síntomas de DP respecto al total de árboles presentes en cada clase diamétrica.

las ciudades (Sjöman, et al., 2012). Con base en lo anterior, nosotros proponemos la hipótesis de que el DP es uno de los mecanismos involucrados en la muerte prematura y, por ello, es de especial relevancia evaluar en qué magnitud acorta la vida media de los árboles y aumenta su tasa de mortalidad. Sin embargo, evaluar esta hipótesis está por fuera del alcance de este artículo.

El hecho de que no existiera relación entre el diámetro medio de las especies y la incidencia de DP (Tabla 1) se explica por las diferencias en el ciclo de vida entre ellas. Por ejemplo, algunas especies alcanzan diámetros máximos pequeños mientras que otras alcanzan grandes tamaños, por lo cual un diámetro medio alto en una especie no necesariamente significa que los individuos sean viejos y viceversa. Los niveles variables de incidencia de DP entre especies, los cuales oscilaron desde muy severo en falso laurel (Ficus benjamina) y chiminango (Pithecellobium dulce) hasta bajo en 13 de las 25 especies estudiadas, denotan tolerancia diferencial a los factores de estrés, lo cual se debe a diferencias ecológicas, fisiológicas y de plasticidad entre ellas; tales diferencias han sido reportadas en otros estudios, incluso entre especies del mismo género (Fraxinus sp.) en el Reino Unido (Percival et al. 2006).

La pregunta que surge entonces es: ¿cuáles rasgos comparten las especies con niveles similares de incidencia de DP? Aunque responder de manera específica esta pregunta no es posible con los datos disponibles, se sabe que las especies difieren en sus requerimientos y tolerancia a las condiciones ambientales y exhiben diversas estrategias fisiológicas y morfológicas que les permiten tolerar o evadir los factores limitantes. Por ejemplo, se ha encontrado que la sobrevivencia bajo condiciones limitantes de suministro de agua en el suelo está determinada principalmente por la resistencia del xilema para generar embolia, lo cual depende fundamentalmente de su anatomía hidráulica y de sus hábitos de defoliación (Benjamin et al., 2005; Tyreet et al., 2003). Entre otras estrategias están la pérdida de hojas, disminución de procesos metabólicos como la fotosíntesis, o cambios en la asignación de biomasa entre órganos (por ejemplo 
desarrollo de raíces profundas como respuesta a la sequía), tamaño foliar, área foliar específica, densidad estomática, diámetro y longitud de los estomas, forma de la copa y orientación de las hojas, entre otras (Pineda-García et al. 2011; Wang et al. 2011). Responder a esta pregunta requiere un seguimiento detallado y periódico de estos y otros rasgos funcionales en las especies estudiadas.

Los mayores valores de incidencia de DP se encontraron en Barbosa y Sabaneta, dos municipios localizados en los extremos norte-sur del Valle de Aburrá, respectivamente; mientras que los valores menores también se encontraron en dos municipios localizados de manera similar en los extremos norte y sur (Girardota y La Estrella); por ello, las diferencias de DP entre municipios no parecen obedecer a factores geográficos, climáticos u otra variable ambiental. En consecuencia, es posible que otras variables no evaluadas como la historia de manejo en cada municipio sean las causantes de las diferencias encontradas.

La mayor probabilidad de ocurrencia de DP en árboles plantados en piso duro y alcorque, en comparación con los de zonas verdes amplias, encontrada en el presente estudio se explica por el efecto combinado del confinamiento de las raíces en un espacio reducido y la compactación del suelo que impide la adecuada aireación y penetración del agua, lo que limita la disponibilidad de oxígeno y agua requeridos por el sistema radical (Meuser, 2010). En concordancia con estos resultados, Koeser et al. (2013) encontraron que los árboles estuvieron más propensos a morir a medida que la amplitud de la zona verde de plantación disminuyó; así mismo, el aumento en el ancho de la zona verde del árbol se correlacionó positivamente con el mejor estado de los árboles. Estos autores también reportan que los árboles tienen mayor probabilidad de morir tempranamente después de la construcción de obras civiles cuando son impactados significativamente por la construcción, y que los árboles adyacentes a tales construcciones tuvieron cerca del doble de probabilidad de morir que aquellos no expuestos.
El modelo también muestra que con el incremento del número de afectaciones a los árboles se incrementa la probabilidad de sufrir DP (Tabla 2), tendencia que mostraron claramente los datos de campo (Figura 5) y que pone en evidencia las consecuencias negativas de intervenciones drásticas y de un manejo deficiente, como también del maltrato y vandalismo a que son sometidos los árboles en los espacios públicos verdes urbanos del Valle de Aburrá (AMVA, 2007). Las afectaciones reportadas en el presente estudio desmejoran la condición del árbol, la cual se ha reportado que aumenta la probabilidad de muerte (Koeser et al., 2013). La presión antrópica por espacio urbano que experimenta este valle (expansión urbana) reduce los espacios verdes para abrir paso al desarrollo de infraestructura de cemento y pavimento (Vélez, 2007) y produce una presión adicional que contribuye al deterioro del arbolado urbano, no solo por la impermeabilización de los suelos alrededor de los individuos, sino también porque incrementa la incidencia de la isla de calor y su efecto sobre este importante y valioso componente de las áreas urbanas. Este fenómeno es particularmente crítico en la ciudad de Medellín, donde los espacios públicos verdes pueden representar entre el $11 \%$ y el $13.9 \%$ de la zona urbana (Sierra, 2012; AMVA, 2007).

Los resultados obtenidos permiten afirmar, con Cooke (2006), que los modelos logísticos tipo logit son estadísticamente adecuados para estudiar el deterioro progresivo y muerte de árboles. Se han usado con éxito para caracterizar, estimar y predecir el comportamiento de la infección y diseminación de la enfermedad Scleroderris, causada por el hongo Gremmeniella abietina (Lagerb.) en plantaciones de pinos en Ontario (Canadá) (Vanier et al., 1998), las devastaciones del Oak (Quercus spp) causada por el fitopatógeno Phytophthora ramorum en California (Meentemeyer et al., 2011) y la enfermedad pitch canker (hongo Fusarium circinatum) en árboles urbanos de Pinus radiata también en California (Storer et al., 2002). En consecuencia, estos modelos son de utilidad 


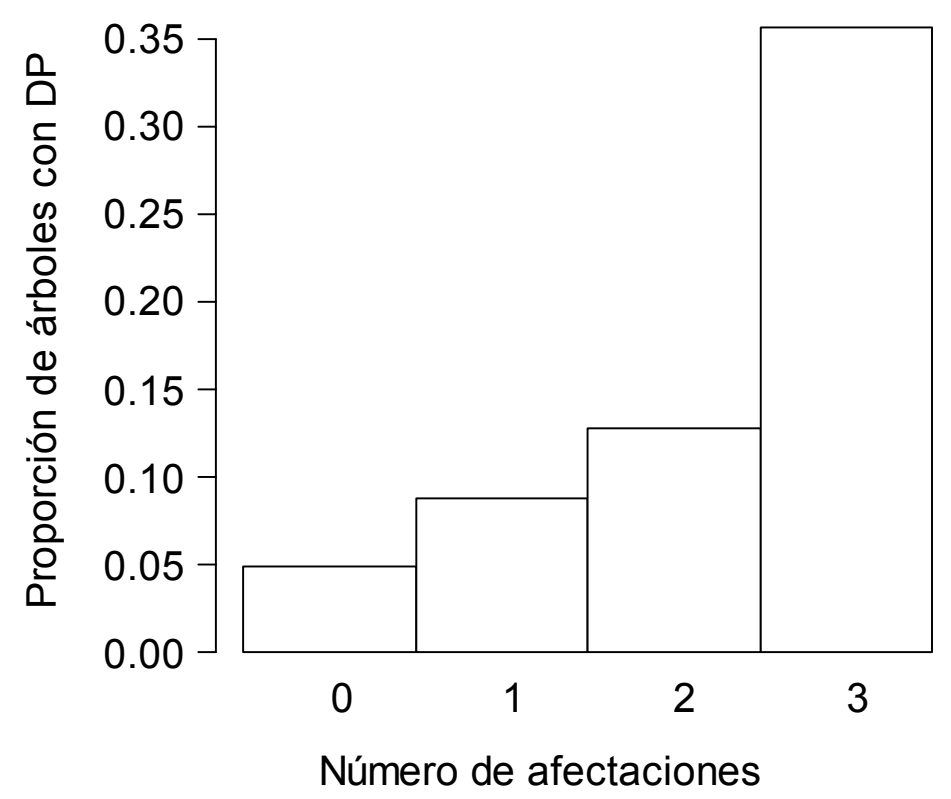

Figura 5. Proporción de árboles con síntomas de DP respecto al número de afectaciones del árbol.

para el diseño de estrategias de manejo dirigidas a minimizar el deterioro y muerte de árboles y sus efectos.

Una consecuencia de especial importancia del DP es la relación que existe entre este y el riesgo que representan los árboles afectados por caída de ramas y/o troncos, no solo para las personas, sino también para los bienes públicos y privados en las ciudades. Por ejemplo, de acuerdo con estadísticas oficiales de la autoridad ambiental de Bogotá (DAMA), 10\% de los árboles representan riesgo inminente por caída de ramas o volcamiento (Tovar, 2007). Los aspectos relacionados con la salud, sobrevivencia y calidad del arbolado urbano son de interés público diverso, no solo para la ciudadanía en general, sino para los planificadores y autoridades ambientales.

\section{CONCLUSIONES}

De acuerdo con los resultados de este estudio, el sitio de siembra, la talla del árbol y el número de afectaciones que ha sufrido un árbol (como podas inadecuadas, heridas en tallo, vandalismo, quemas, anillamiento, presencia de hongos e insectos, etc.) inciden en el DP y varía entre especies; estos resultados refuerzan la idea de que el DP es producto de la interacción de causas naturales y antrópicas, las cuales afectan directa o indirectamente la fisiología y salud de los árboles en los espacios urbanos. Los resultados acá presentados brindan herramientas para la gestión del arbolado urbano y para el desarrollo de políticas públicas relacionadas con su planificación y manejo, que permitan maximizar los beneficios ambientales de este importante componente del paisaje urbano con base en criterios de sostenibilidad ambiental, económica y social. Por ejemplo, es claro que la adaptación exitosa y vigor en las condiciones ambientales adversas de la ciudad es un criterio que debería tener más peso en la selección de árboles para plantar en las zonas verdes urbanas. Así mismo, el manejo integral del arbolado urbano, mediante el cual se disminuyan las causas de afectación de origen antrópico y se realicen actividades que mejoren el vigor y la sanidad de los árboles, es un componente fundamental de 
las medidas dirigidas a enfrentar el problema del DP. Lo anterior implica contar con un sistema de monitoreo periódico del estado fitosanitario del arbolado urbano y de actualización permanente de la base de datos georreferenciada del mismo, así como de acción inmediata para prevenir y corregir eventuales problemas.

\section{AGRADECIMIENTOS}

Esta investigación se realizó mediante el convenio 471 de 2012 entre la Universidad Nacional de Colombia, Sede Medellín y Área Metropolitana del Valle de Aburrá. Agradecimientos especiales al equipo técnico del proyecto y a todas las personas que contribuyeron al desarrollo de la presente investigación.

\section{REFERENCIAS BIBLIOGRÁFICAS}

Área Metropolitana del Valle de Aburrá (AMVA), Corporación Autónoma Regional de Centro de Antioquia (Corantioquia), \& Universidad Nacional de Colombia (UNAL). (2007). Plan de ordenación y manejo de la cuenca del río Aburrá. Medellín. 189 p. Recuperado de http://issuu.com/cornare/docs/ pomca_rio_aburra

Área Metropolitana del Valle de Aburrá (AMVA), Escuela de Ingeniería de Antioquia (EIA), \& Universidad Nacional de Colombia (UNAL). (2010). Evaluación y cuantificación de la muerte súbita de árboles en las zonas verdes urbanas del Valle de Aburrá. Convenio CA 380 de 2010. 113 p.

Área Metropolitana del Valle de Aburrá (AMVA), \& Universidad Nacional de Colombia (UNAL). (2014). Aunar esfuerzos técnicos y económicos para el desarrollo e implementación de un plan de manejo integral para el arbolado urbano del Valle de Aburá con énfasis en intervenciones para el control de la muerte súbita. Convenio CD 471 de 2012. 457 p

Área Metropolitana del Valle de Aburrá (AMVA). (2007). Plan maestro de espacios públicos verdes urbanos (resumen). Recuperado de http://www. areadigital.gov.co:9000/ZonasVerdes/Paginas/Publicaciones.aspx

Benjamin, K., Domon, G. \& Bouchard, A. (2005). Vegetation composition and succession of abandoned farmland: effects of ecological, historical and spatial factors. Landscape Ecology, 20, 627-647.

Cardona, M. (2006). Ordenamiento y manejo integral del territorio metropolitano del Valle de Aburrá, con énfasis en el recurso agua. Producción + Limpia, 1(2), 9-27.

Cooke, B.M. (2006). Disease assessment and yield loss. En B. M. Cooke, D. G. Jones, \& B. Kaye (eds.), The epidemiology of plant diseases (pp. 43-80). Netherlands: Springer.

Corporación Autónoma Regional de Centro de Antioquia (Corantioquia). (1997). Plan de gestión ambiental regional 1998-2006. Medellín: Imprenta Departamental de Antioquia. Recuperado de http://www.corantioquia.gov.co/docs/PATSUP.htm

Cregg, B., \& Dix, M. (2001). Tree moisture stress and insect damage in urban areas in relation to heat island effects. Journal of Arboriculture, 27(1), 8-17.

Dale, V., Joyce, L., McNulty, S., Neilson, R., Ayres, M., Flannigan, M., Hanson, P., Irland, L., Lugo, A.E., Peterson, C., Simberloff, D., Swanson, F., Stocks, B., \& Wotton, M. (2001). Climate change and forest disturbances. BioScience, 51, 723-734.

Dale, V., Tharp, M.L., Lannom, K., \& Hodges, D.G. (2010). Modeling transient response of forest to climate change. Science of the Total Environment, 408, 1888-1901.

Del Valle, J. I. (2002). Mortality as a mathematical function of organic growth and diameter structure. Plant Biosystems, 136(2), 217-221,

Franklin, J.F., Shugart, H.H., \& Harmon, M.E. (1987). Tree death as an ecological process: The causes, consequences, and variability of tree mortality. BioScience, 37(8), 550-556.

Garbelotto, M., \& Schmidt, D. (2009). Phosphonate controls sudden oak death pathogen for up to 2 years. California Agriculture, 63, 10-17.

Griliches, Z., \& Intriligator, M. (1994). Handbook of Econometrics, Vol 2. New York: Elsevier. 1457 p. 
Guo, Q., Kelly, M., \& Graham, C. (2005). Support vector machines for predicting distribution of Sudden Oak Death in California. Ecological Modelling, 182, 75-90.

Holdridge, L.R. (1996). Ecología basada en zonas de vida. Instituto Interamericano de Cooperación para la Agricultura. $216 \mathrm{p}$.

Koeser, A., Hauer, R., Norris, K., and Krouse, R. (2013). Factors influencing long-term street tree survival in Milwaukee, WI, USA. Urban Forestry \& Urban Greening, 12, 562-568.

Lacan, I., \& McBride, J. R. (2008). Pest vulnerability matrix (PVM): a graphic model for assessing the interaction between tree species diversity and urban forest susceptibility to insects and diseases. Urban Forestry \& Urban Greening, 7, 291-300.

Lugo, A.E., \& Scatena, F.N. (1996). Background and catastrophic tree mortality in tropical moist, wet, and rain forests. Biotropica, 28(4), 585-599.

Manion, P.D. (1991). Tree Disease Concepts ( $\left.2^{\text {nd }} e d.\right)$. Prentice Hall, Engelwood Cliffs, NJ. 399 p.

Meentemeyer, R.K., Cunniffe, N.J., Cook, A.R., Filipe, J.A., Hunter, R.D., Rizzo, D.M., \& Gilligan, C.A. (2011). Epidemiological modeling of invasion in heterogeneous landscapes: spread of sudden oak death in California (1990-2030). Ecosphere, 2(2), 1-24.

Meuser H. (2010). Contaminated urban soils. Springer, Heildelberg. 320 p.

Miller, R.H., \& Miller, R.W. (1991). Planting survival of selected street tree taxa. Journal of Arboriculture, 17, 185-191.

Percival, G.C., Keary I.P., \& AL-Habsi, S. (2006). An assessment of the drought tolerance of Fraxinus genotype for urban landscape planting. Urban Forestry \& Urban Greening, 5(1):17-27.

Pineda-García, F. Paz, H., \& Tinoco-Ojanguren, C. (2011). Morphological and physiological differentiation of seedlings between dry and wet habitats in a tropical dry forest. Plant, Cell and Environment, 34, 1536-1547.

R Core Team. (2013). R: A language and environment for statistical computing. Vienna, Austria. R Fundation for Statistical Computing. ISBN 3-900051-070, recuperado de http://www.R-project.org.
Rizzo, D., \& Garbelotto, M. (2003). Sudden oak death: endangering California and Oregon forest ecosystems. Frontiers in Ecology and the Environment, 1, 197-2004.

Salisbury, F.B., \& Ross, C.W. (1985). Plant physiology $\left(3^{\text {rd }}\right.$ ed.). Wadsworth, Belmont, California. $540 \mathrm{p.}$

Santamour Jr., F. S. (2004). Trees for urban planting: diversity uniformity, and common sense. En C. Elevitch, The Overstory Book: Cultivating connections with trees (pp. 396-399). Holualoa, Hawaii: Permanent Agriculture Resources.

Sierra, M. (2012). Ciudad y fauna urbana: Un estudio de caso orientado al reconocimiento de la relación hombre, fauna y hábitat urbano en Medellín (Tesis de Maestría en Estudios Urbano-Regionales). Medellín: Universidad Nacional de Colombia. 66 p.

Sjöman, H., Gunnarsson, A., Pauleit, S., \& Bothmer, R. (2012). Selection Approach of Urban Trees for Inner City Environments: Learning from Nature. Arboriculture and Urban Forestry, 38(5), 194-204.

Spittlehouse, D., \& Stewart, R. (2003). Adaptation to climate change in forest management. BC Journal of Ecosystems and Management, 4, 1-11.

Storer, A.J., Wood, D.L., \& Gordon, T.R. (2002). The epidemiology of Pitch Canker of Monterey pine in California. Forest Science, 48(4), 694-700.

Sturrock, R.N., Frankel, S.J., Brown, A.V., Hennon, P.E., Kliejunas, J.T., Lewis, K.J., Worrall, J.J., \& Woods, A.J. (2011). Climate change and forest diseases. Plant Pathology, 60, 133-149.

Tovar, G. (2007). Manejo del arbolado urbano en Bogotá. Territorios, 16-17, 149-174.

Tyree M.T. Engelbrecht, B.M.J., Vargas, G. \& Kursar, T. (2003). Desiccation tolerance of five tropical seedlings in Panama. Relationship of field assessment of drought performance. Plant Physiology, 132, 1439-1447.

Vanier, L.A., Hopkin, A., McKenney, D., \& Wang, Y. (1998). A spatial, climate-determined risk rating for Scleroderris disease of pines in Ontario. Canadian Journal of Forest Research, 28, 1398-1404.

Vélez, L.A. (2007). Paisajismo y ecología del paisaje en la gestión de la arborización de calles. Una 
referencia a la ciudad de Medellín, Colombia. Investigación, 10(4), 131-140.

Walpole R.E., \& Myers, R. (1992). Probabilidad y estadística. México: McGraw Hill. 797 p.

Wang, J.C., Shi, X., Zhang, D.Y., \& Yin, L.K. (2011). Phenotypic plasticity in response to soil moisture availability in the clonal plant Eremosparton songoricum (Litv.) Vass. Journal of Arid Land 3(1), 34-39.
Waring, R.H. (1987). Characteristics of trees predisposed to die: Stress causes distinctive changes in photosynthate allocation. BioScience, 37(8), 569-574.

Wooldridge, M.J. (2002). Econometric analysis of cross section and panel data. Cambridge: Massachusetts Institute of Technology. 752 p.

Yepes, A.P., Del Valle, J.I., Jaramillo, S.L., \& Orrego, S.A. (2010). Recuperación estructural en bosques sucesionales andinos de Porce. Revista de Biología Tropical, 58(1), 427-445. 